\title{
Sliding Mode Repetitive Control of PWM Voltage Source Inverter
}

\author{
Sufen Chen, Y. M. Lai, Siew-Chong Tan, and Chi K. Tse \\ Department of Electronic and Information Engineering, The Hong Kong Polytechnic University, Hong Kong, China
}

\begin{abstract}
This paper proposes a hybrid sliding mode repetitive control scheme that combines both the features of the sliding mode control and the repetitive control to achieve excellent transient and steady-state system performances in voltage source inverters. The principle of equivalent control is adopted to integrate the two methodologies and to facilitate the design and analysis of the proposed scheme. A specific low pass filter is also introduced to improve the transient response of the regulation. Experimental results show that fast dynamical responses are achieved through the sliding mode control, while low harmonic distortions are achieved through the repetitive control.
\end{abstract}

Index Terms - Repetitive control, sliding mode control, THD, voltage source inverter.

\section{INTRODUCTION}

The pulse-width modulation (PWM) voltage source inverter (VSI) is extensively used in AC power conditioning systems. Many of such systems require the VSI to have a fast dynamical response, an accurate output regulation, and a low total harmonic distortion (THD), even when the operating load is highly nonlinear and/or distorted. Currently, most of these systems in the industry use the conventional type of linear PWM controllers, which are simple and cost effective for common applications that do not require a tight regulation standard. However, for applications requiring more stringent regulation, nonlinear control methodologies have to be employed. One such methodology is the sliding mode control (SMC) [1] [3]. It has been shown that the adoption of SMC in the VSI can significantly improve the transient characteristic as well as the robustness of the VSI. However, in terms of the steadystate performance, the use of SMC in VSI cannot satisfactorily alleviate the THD. The presence of harmonic distortion is solely attributed to the imperfect tracking of the sinusoidal varying reference signal. This is a result of having only limited DC gain in the SM controller. Thus, by similar reasoning, most other control methodologies are incapable of fully containing this THD issue.

Theoretically, the alleviation of the THD would mean that the steady-state tracking of the periodic signal must be very accurate. To do this, control approaches specialized for achieving precise tracking of periodic signal have to be incorporated. One approach commonly chosen for this purpose is the repetitive control (RC) [4], [5], which is based on the internal model principle [6]. The basic operating principle of the $\mathrm{RC}$ is to observe the system's external periodic signals for one cycle period, and then to generate in the next cycle period a corresponding compensating signal that ensures precise tracking of the external signals [5]. The recent applications of the RC in the VSI to alleviate the THD are reported in [7], [8]. It has been demonstrated that a very low THD can be obtained using this control methodology. However, the works also reveal that due to its one-cycle-delay requirement, the $\mathrm{RC}$ is a relatively slow-responding control technique when dealing with instantaneous transient changes. This makes it inappropriate for VSI that requires a fast dynamical response, unless the RC is incorporated along with a fast responding nonlinear control scheme. This implies a hybrid controller that simultaneously achieves the various objectives of having fast response, accurate regulation, and very low THD.

Following this direction, we propose in this paper a hybrid sliding mode repetitive control (SMRC) scheme that combines the features of SMC and RC to offer excellent transient and steady-state performances to the VSI. Note that previous works on the SMC of power converters are mainly performed in the time domain [9], [10], whereas the work on RC is performed in the frequency domain [8], [11]. Thus, this paper serves to clarify the various issues concerning the combined application of both control methodologies, to provide guidelines towards their theoretical design, and to give experimental verification and evaluation of the proposed controller.

\section{State-Space Model of Voltage Source INVERTER}

Fig. 1 shows a typical single phase inverter system. The load to the inverter can either be resistive or wave-rectified depending on the nature of the application. Using the notation described in the figure, the state-space representation of the

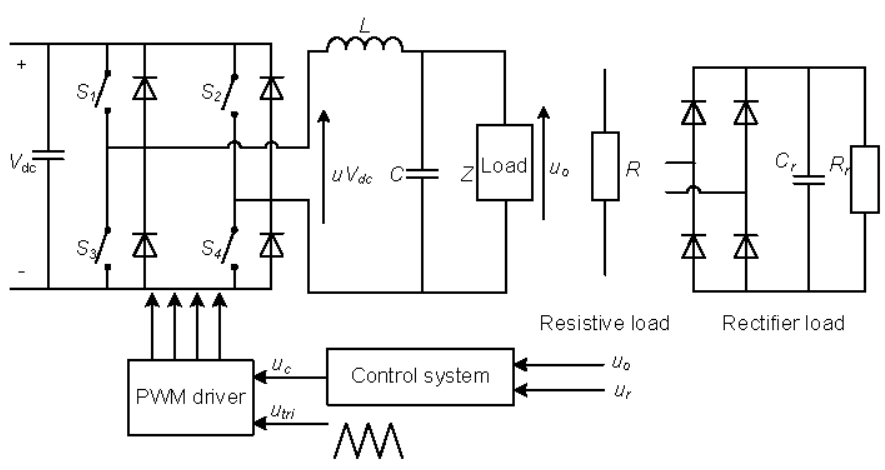

Fig. 1. Single-phase inverter system. 
inverter dynamics in terms of its PID voltage errors can be written as

$$
\dot{\boldsymbol{x}}=A \boldsymbol{x}+B u+D
$$

where

$$
\begin{aligned}
\boldsymbol{x} & =\left[\begin{array}{l}
x_{1} \\
x_{2} \\
x_{3}
\end{array}\right]=\left[\begin{array}{c}
u_{r}-u_{o} \\
\dot{u}_{r}-\dot{u}_{o} \\
\int\left(u_{r}-u_{o}\right) d t
\end{array}\right], \\
A & =\left[\begin{array}{ccc}
0 & 1 & 0 \\
-\frac{1}{L C} & -\frac{1}{Z C} & 0 \\
1 & 0 & 0
\end{array}\right], \\
B & =\left[\begin{array}{c}
0 \\
-\frac{V_{d o}}{L C} \\
0
\end{array}\right], \\
D & =\left[\begin{array}{c}
0 \\
\ddot{u}_{r}+\frac{\dot{u}_{r}}{Z C}+\frac{u_{r}}{L C} \\
0
\end{array}\right] ;
\end{aligned}
$$

and $u_{r}, V_{d c}$, and $u_{o}$ are the sinusoidal reference voltage,

the input voltage, and the output voltage, respectively; $C, L$, and $Z$ are the capacitance, inductance, and load impedance, respectively; and $u \in\{-1,+1\}$ is the control signal, which respectively generates an input voltage of $-V_{d c}$ and $+V_{d c}$ at the input of the $L C$ filter. If we adopt an amplitude modulation ratio of $m_{a}=\hat{u}_{c}(t) / \hat{u}_{t r i}<1$, where $u_{c}$ is the compensated control signal with amplitude $\hat{u}_{c}(t)$, and $\hat{u}_{t r i}$ is the amplitude of the PWM triangular carrier, then the averaged time-continuous value of the discrete control signal $u$ will be

$$
\bar{u}=\frac{u_{c}}{\hat{u}_{\text {tri }}},
$$

where $\bar{u}$ is the averaged duty cycle bounded by positive and negative unity. Choosing $\hat{u}_{t r i}=1$, the averaged duty cycle will be simplified to $-1<\bar{u}=u_{c}<+1$.

\section{Proposed Control Methodology}

\section{A. Sliding Mode Control}

The principle of SMC and its application to power electronics can be found in [1]-[3], [9] and [10]. In this work, the commonly used PID SM voltage control structure is adopted for investigation. The switching control law for this structure is given as

$$
\begin{cases}u=+1, & \text { for } S>0 \\ u=-1, & \text { for } S<0\end{cases}
$$

where sliding function

$$
S=\alpha_{1} x_{1}+\alpha_{2} x_{2}+\alpha_{3} x_{3}=\boldsymbol{J}^{\mathrm{T}} \boldsymbol{x}
$$

with sliding coefficients $\boldsymbol{J}^{\mathrm{T}}=\left[\begin{array}{lll}\alpha_{1} & \alpha_{2} & \alpha_{3}\end{array}\right]$. According to SMC theory, the existence condition of a system working in sliding mode operation can be found by using Lyapunov's direct method to solve $S \dot{S}<0$. By substituting (1), (3) and (4), the existence condition is given by

$\left\{\begin{array}{l}\left(-\frac{1}{L C}+\frac{\alpha_{3}}{\alpha_{2}}\right) x_{1}+\left(\frac{\alpha_{1}}{\alpha_{2}}-\frac{1}{Z C}\right) x_{2}-\frac{V_{d_{o}}}{L C}+\ddot{u}_{r}+\frac{\dot{u}_{r}}{Z C}+\frac{u_{r}}{L C}<0 \\ \left(-\frac{1}{L C}+\frac{\alpha_{3}}{\alpha_{2}}\right) x_{1}+\left(\frac{\alpha_{1}}{\alpha_{2}}-\frac{1}{Z C}\right) x_{2}+\frac{V_{d o}}{L C}+\ddot{u}_{r}+\frac{i_{r}}{Z C}+\frac{u_{r}}{L C}>0\end{array}\right.$.

In sliding mode operation, the dynamics of the system can be characterized using the invariance conditions $S=0$ and
$\dot{S}=0$. With the condition $S=0$, the system is represented as a second order homogeneous differential equation. A set of design equations for the under-studied inverter control system can be obtained using the approach described in [10]. Note that the selection of the sliding coefficients should comply with the stability condition of the second order system and the existence condition (5) in the sliding mode operation. With the condition $\dot{S}=0$, i.e., $\boldsymbol{J}^{\mathrm{T}} A \boldsymbol{x}+\boldsymbol{J}^{\mathrm{T}} B u_{e q}+\boldsymbol{J}^{\mathrm{T}} D=0$, the equivalent control signal, which is the averaged value of the control signal $u$, can be obtained as

$$
\begin{aligned}
u_{e q}= & -\left[\boldsymbol{J}^{\mathrm{T}} B\right]^{-1} \boldsymbol{J}^{\mathrm{T}}(A \boldsymbol{x}+D) \\
= & \frac{L C}{V_{d c}}\left(\frac{\alpha_{3}}{\alpha_{2}}-\frac{1}{L C}\right)\left(u_{r}-u_{o}\right)+\frac{L C}{V_{d c}}\left(\frac{\alpha_{1}}{\alpha_{2}}-\frac{1}{Z C}\right)\left(\dot{u}_{r}\right. \\
& \left.-\dot{u}_{o}\right)+\frac{L C}{V_{d c}}\left(\ddot{u}_{r}+\frac{\dot{u}_{r}}{Z C}+\frac{u_{r}}{L C}\right)
\end{aligned}
$$

where $-1<u_{e q}<+1$. Since $u_{e q}$ is the equivalent control of sliding mode, which directly controls the inverter, it is reasonable to relate $u_{e q}$ with the averaged duty cycle $\bar{u}$ in (2) to establish the relationship $u_{e q}=\bar{u}=u_{c}$. For inverter system working in steady state, both the input voltage $V_{d c}$ and the load impedance $Z$ are static, so equation (6) is linear, and it can be written in Laplace form as

$$
\begin{aligned}
U_{e q}(s)= & U_{c}(s) \\
= & {\left[\frac{L C}{V_{d c}}\left(\frac{\alpha_{1}}{\alpha_{2}}-\frac{1}{Z C}\right) s+\frac{L C}{V_{d c}}\left(\frac{\alpha_{3}}{\alpha_{2}}-\frac{1}{L C}\right)\right]\left[U_{r}(s)\right.} \\
& \left.-U_{o}(s)\right]+\left(\frac{L C}{V_{d c}} s^{2}+\frac{L}{V_{d c} Z} s+\frac{1}{V_{d c}}\right) U_{r}(s)(7)
\end{aligned}
$$

under the assumption that the initial condition is nulled. This transformation to the $s$ domain is critical for combining SMC with RC. Fig. 2(a) illustrates the block diagram of an inverter with the derived sliding mode controller given in (7). Here, the tracking error is given by

$$
E_{0}(s)=\frac{U_{r}(s)-D(s)-U_{r}(s) G_{s 2}(s) G_{p}(s)}{1+G_{s 1}(s) G_{p}(s)},
$$

where $G_{s 1}(s)=\frac{L C}{V_{d o}}\left(\frac{\alpha_{1}}{\alpha_{2}}-\frac{1}{Z C}\right) s+\frac{L C}{V_{d o}}\left(\frac{\alpha_{3}}{\alpha_{2}}-\frac{1}{L C}\right)$ and $G_{s 2}(s)=$ $\frac{L C}{V_{d o}} s^{2}+\frac{L}{V_{d_{0}} Z} s+\frac{1}{V_{d o}} ; G_{p}(s)$ denotes the inverter plant; and $D(s)$ denotes the external disturbances.

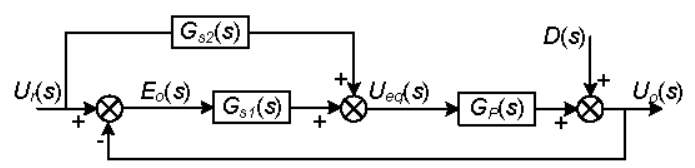

(a)

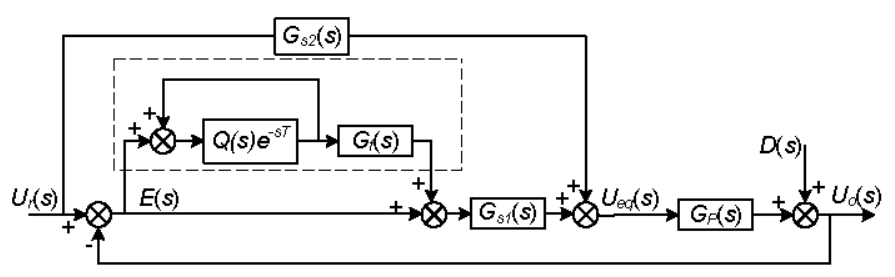

(b)

Fig. 2. Block diagram of: (a) the SMC, (b) the proposed SMRC. 


\section{B. Repetitive Control}

The detailed description of $\mathrm{RC}$ and its applications to power electronics can be found in [4]-[8], [11] and [12]. Fig. 2(b) shows the block diagram of the proposed SMRC scheme with the inverter plant. It is obtained from the SMC system described in Fig. 2(a) by incorporating a plug-in RC component into the system.

1) Periodic Error Elimination: The tracking error of the SMRC system shown in Fig. 2(b), in terms of $E_{0}(s)$ of the SMC system, is given by:

$$
E(s)=E_{0}(s) \times \frac{1-Q(s) e^{-s T}}{1-\left[1-G_{f}(s) H(s)\right] Q(s) e^{-s T}},
$$

where $H(s)=\frac{G_{s 1}(s) G_{p}(s)}{1+G_{s 1}(s) G_{p}(s)}$ and $T$ is the period of the reference signal.

Assuming that the original SMC system is asymptotically stable and that

$$
\left|\left[1-G_{f}(s) H(s)\right] Q(s)\right|<1,
$$

where $s=j \omega$, for all $\omega$, the stability condition of SMRC system is satisfied (small gain theorem [13]). Equation (9) can be expressed as $E(s)=E_{0}(s)\left\{1-G_{f}(s) H(s) Q(s) e^{-s T}-\right.$ $G_{f}(s) H(s)\left[1-G_{f}(s) H(s)\right] Q^{2}(s) e^{-2 s T}-G_{f}(s) H(s)[1-$ $\left.\left.G_{f}(s) H(s)\right]^{2} Q^{3}(s) e^{-3 s T}+\cdots\right\}$. Theoretically, by setting $1-G_{f}(s) H(s)=0$ and $1-Q\left(j k \omega_{0}\right) e^{-j k \omega_{0} T}=0, k=$ $0,1,2, \cdots$, where $\omega_{0}=2 \pi / T$, we can ensure that the steadystate tracking error converges to zero at the occurrence of every harmonic. However, due to the non-idealities of the system, such an ideal design condition is practically impossible. Instead, it is more appropriate to consider the design condition:

$$
1-G_{f}(s) H(s) \approx 0 \text { and } 1-Q\left(j k \omega_{0}\right) e^{-j k \omega_{0} T} \approx 0,
$$

and for harmonics limited to a certain frequency bandwidth.

2) Selection of Repetitive Control Parameters: It is easy to understand from (11) that the parameter $G_{f}(s)$ should be a zero-magnitude-and-phase compensator for $H(s)$. An intuitive way to design $G_{f}(s)$ is to make $G_{f}(s)=1 / H(s)$. However, due to system parameters' drifts (e.g. capacitor's ESR variation), the use of this design will result in a system which is stable at low frequencies, but unstable at high frequencies. Hence, to tackle this issue, we propose to include an additional high-frequency pole to the compensator $G_{f}(s)$ to remove the high frequency signals that will cause the instability. This makes

$$
G_{f}(s)=\frac{1}{H(s)} \times \frac{p_{1}}{\left(s+p_{1}\right)},
$$

where $p_{1}$ is a high frequency pole. This will not affect the low frequency characteristic of $G_{f}(s)$. Additionally, careful observation of (11) reveals that $Q(s)$ should be unity while (10) indicates that $Q(s)$ should be less than unity at high frequencies because $1-G_{f}(s) H(s)$ tends to unity as $G_{f}(s) H(s) \rightarrow 0$ at high frequencies. Thus, $Q(s)$ should be a low pass filter with unity magnitude and zero phase shift at low frequencies, and slightly lower than " 1 " at high frequencies to ensure system stability. To satisfy the imposing requirements, a low pass filter:

$$
Q(s)=\frac{e^{\tau s}}{\frac{s^{2}}{\omega_{q}^{2}}+\frac{2 \zeta s}{\omega_{q}}+1},
$$

as given in [12] is adopted. Here, the damping ratio is chosen as $\zeta=0.707$ to give a flat magnitude with quasilinear phase shift characteristic within its bandwidth. The time advance $\tau$ is selected to equal the effective time delay of the denominator term, i.e., $2 \zeta / \omega_{q}$. This ensures that the low pass filter achieves a zero phase shift at low frequencies. The selection of bandwidth $\omega_{q}$ is influenced by the pre-designed system and $G_{f}(s)$. A larger value of $\omega_{q}$ improves tracking accuracy, but degrades the system stability. The appropriate $\omega_{q}$ can be determined through simulation and experimental tuning.

\section{Reduction of the Dynamic Sensitivity of RC}

As mentioned before, the operating characteristic of RC is that it uses information in the current period to generate a corresponding control signal for the next cycle period. This results in a one cycle-period time delay in the control action, which in the event of a disturbance, will introduce a distortion to the transient behavior of the system. This is because the disturbance observed by the $\mathrm{RC}$ will be applied to influence the system performance only one cycle-period after its occurrence. However, at that point in time, the disturbance would have already been corrected by the SMC component in the controller. Hence, the correction enforced by the RC can be regarded as a form of parasitic distortion. Note that the $\mathrm{RC}$ does not hinder the fast response of the SMC component since there is a direct channel from the feedback to the input of $G_{s 1}(s)$ without passing through the RC (see Fig. 2(b)). To resolve the aforementioned problem, we propose to incorporate a specific low pass filter (SLPF):

$$
L(s)=\frac{p_{2}}{s+p_{2}},
$$

with bandwidth $p_{2}$, to the $\mathrm{RC}$ component to filter out the high frequency disturbances of the system, i.e., to reduce its sensitivity to dynamics introduced by abrupt changes. Recall that there is already an existing frequency pole $p_{1}$ in $G_{f}(s)$ (see (12)). The inclusion of the SLPF means that there will be an overlap between $p_{1}$ and $p_{2}$. Hence, it is sufficient to consider only the filter with the lower cutoff frequency for the design. Since the proposed SLPF is typically of a much lower bandwidth, i.e., $p_{2}<p_{1}$, it is sufficient to consider only the SLPF for the design of the RC component. With the filter related to $p_{1}$ removed, the SLPF filter assumes both the roles of ensuring system robustness and reducing the sensitivity of $\mathrm{RC}$.

\section{RESUlTS AND Discussions}

An experimental system has been setup to verify the proposed SMRC using dSPACE DS1103 [14]-[16] and Matlab/Simulink to control the IGBT switches in the VSI illustrated in Fig. 1. The calculation step size of DS1103 is set at $15 \mu \mathrm{s}$. The sampling rate of the A/D converter is $f_{s p}=66.7$ 


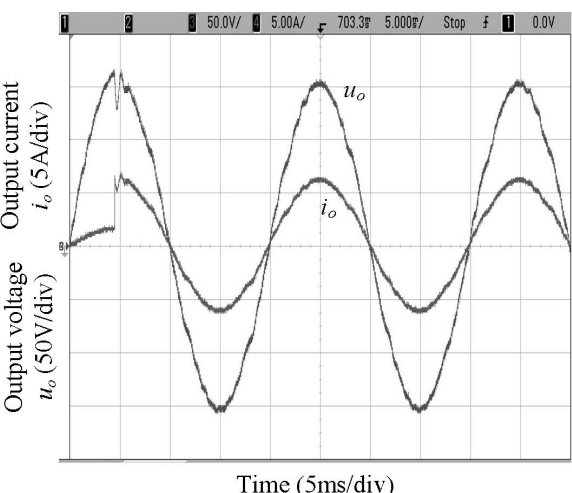

(a)

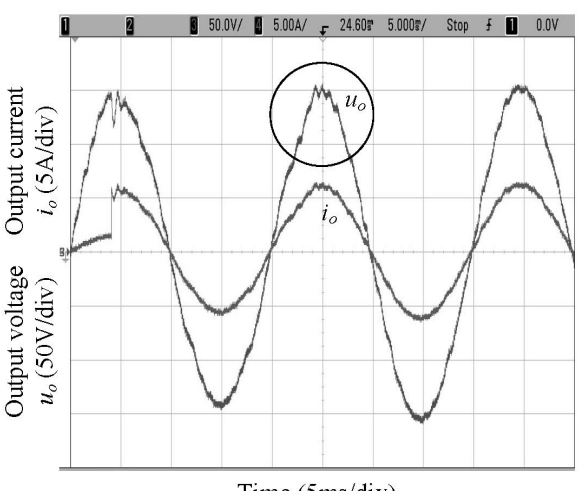

(b)

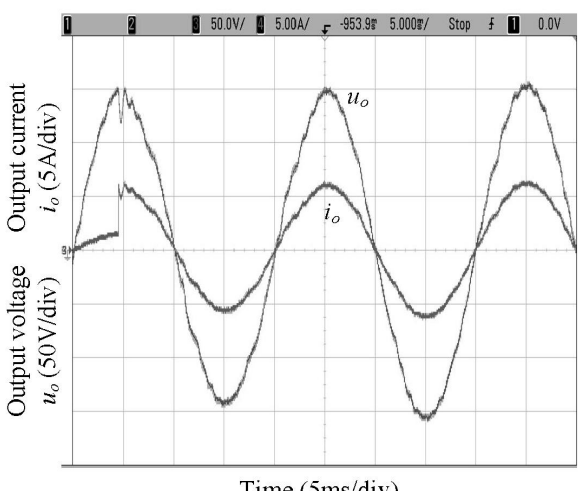

(c)

Fig. 3. The output voltage $u_{o}(50 \mathrm{~V} / \mathrm{div})$ and output current $i_{o}$ (5A/div) at step load change under (a) SMC; (b) SMRC; and (c) SMRC with SLPF.

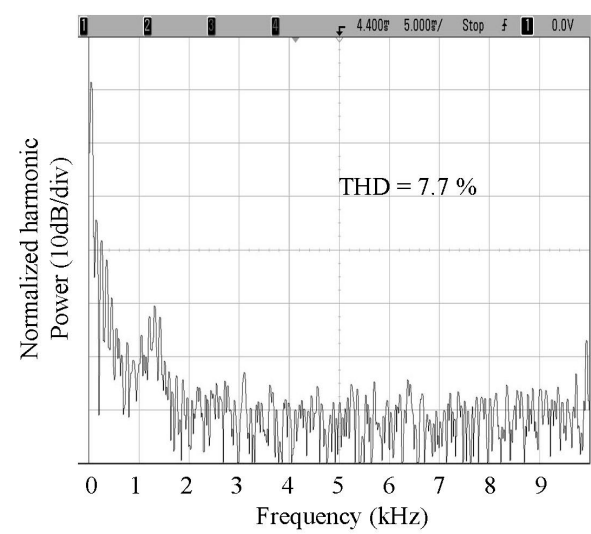

(a)

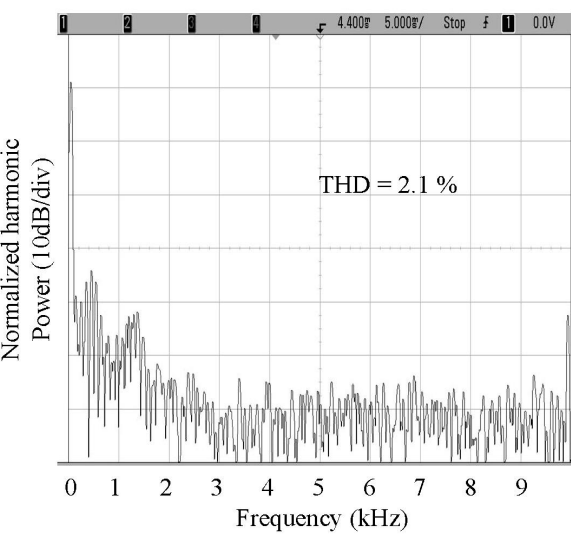

(b)

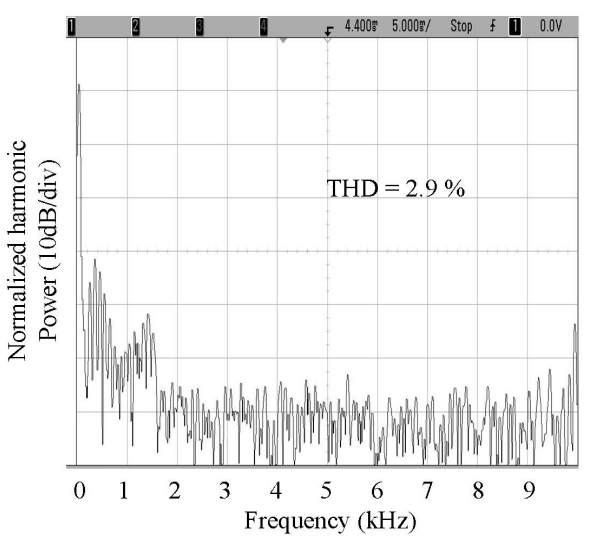

(c)

Fig. 4. The frequency spectrum of inverter output voltage under rectifier load with (a) SMC; (b) SMRC; and (c) SMRC with SLPF,

$\mathrm{kHz}$ and the PWM switching frequency is $f_{s w}=10 \mathrm{kHz}$, so the oversampling rate of the system is $f_{s p} / f_{s w}=6.67$. The system parameters are given as $V_{d c}=250 \mathrm{Vdc} ; U_{r}=110$ $\mathrm{V}_{(\mathrm{rms})}, 50 \mathrm{~Hz} ; L=1.5 \mathrm{mH}$ and $C=20 \mu \mathrm{F}$. The controller is designed with $\frac{\alpha_{1}}{\alpha_{2}}=5.33 \times 10^{3}, \frac{\alpha_{3}}{\alpha_{2}}=4.267 \times 10^{7} ; p_{1}=$ $100 \mathrm{krad} / \mathrm{s}$ with $\omega_{q}=2500 \mathrm{rad} / \mathrm{s}$; and $p_{2}=10 \mathrm{krad} / \mathrm{s}$ with $\omega_{q}=$ $1500 \mathrm{rad} / \mathrm{s}$.

\section{A. Dynamic Performance}

The dynamic performance of the system is tested using a resistive load with step change from $1 / 4$ load $(121 \mathrm{~W})$ to full load $(484 \mathrm{~W})$. Fig. 3 shows the corresponding waveforms of the output voltage $u_{o}$ and the output current $i_{o}$ under SMC, SMRC, and SMRC with SLPF. From the figure, it can be seen that the dynamical responses of all three controllers are similarly optimal at the instance of the step load change. This is credited to the excellent dynamic property of the SMC. On the other hand, the excellent consistency of the dynamical responses also verifies that the $\mathrm{RC}$ does not alter the dynamical response capability of the SMC towards handling the transient disturbance. This confirms the discussion of the previous section. However, in the cycle-period following the disturbance, it is observed that while the regulations of the inverter with SMC (Fig. 3(a)) and SMRC with SLPF (Fig. $3(\mathrm{c})$ ) are returned to normal, the regulation with SMRC (Fig. 3(b)) is distorted. As previously discussed, this is caused by the one cycle delay of the RC action. The results concurrently verify that the inclusion of SLPF can alleviate the parasitic distortion from the regulation.

\section{B. Steady-State Performance}

The steady-state performance of the proposed control scheme is tested using a rectifier load with $C_{r}=330 \mu \mathrm{F}$ and $R_{r}=50 \Omega$. Fig. 4 shows the harmonic contents in the output voltage of the inverter under SMC, SMRC, and SMRC with SLPF. A comparison of the three graphs shows that the THD of SMRC is the lowest (2.1\%), followed by SMRC with SLPF (2.9\%), and then SMC (7.7\%). This shows that the use of SLPF can affect the tracking accuracy of the RC component. Yet, without RC, the use of SMC alone will give a high THD level which may not meet the required regulation standard.

\section{CONCLUSION}

A sliding mode repetitive control scheme is proposed for the PWM voltage source inverter to achieve excellent dynamic and steady-state performances. The sliding mode and repetitive control methodologies are combined into a single control scheme using the equivalent control principle. The design of the control scheme for both the sliding mode and the repetitive 
control components have been discussed in the paper. It is demonstrated that a specific low pass filter can be incorporated into the scheme to reduce the system's dynamical sensitivity towards abrupt changes. This can improve the overall transient response of the system. Finally, it can be concluded from the experimental results that both the sliding mode control and the repetitive control method can be combined into a hybrid control scheme that inherits the respective advantages of the individual schemes to achieve excellent control performances in the inverter system.

\section{ACKNOWLEDGMENT}

The authors would like to thank Mr. S. Y. Lam for help in developing the experimental prototype.

\section{REFERENCES}

[1] J. J. E. Slotine and W. Li, "Sliding control," in Applied Nonlinear Control. Englewood Cliffs, NJ: Prentice-Hall, 1991, ch. 7.

[2] M. Carpita and M. Marchesoni, "Experimental study of a power conditioning system using sliding mode control," IEEE Trans. Power Electron., vol. 11, no. 5, pp. 731-742, Sep. 1996.

[3] R. Gupta and A. Ghosh, "Frequency-domain characterization of sliding mode control of an inverter used in DSTATCOM application," IEEE Trans. Circuits Syst. I, vol. 53, no. 3, pp. 662-676, Mar. 2006.

[4] M. Nakano and S. Hara, "Microprocessor-based repetitive control," in Microprocessor-Based Control Systems, N. K. Sinha, Ed. Dordrecht: Reidel Publ. Co., 1986.
[5] W. S. Yao and M. C. Tsai, "Analysis and estimation of tracking errors of plug-in type repetitive control systems," IEEE Trans. Automat. Contr., vol. 50, no. 8, pp. 1190-1195, Aug. 2005.

[6] B. A. Francis and W. M. Wonham, "The internal model principle for linear multivariable regulators," Appl. Math. Opt., vol. 2, no. 2, pp. 170194, 1975.

[7] Y. Q. Ye, K. L. Zhou, B. Zhang, D. W. Wang, and J. C. Wang, "Highperformance repetitive control of PWM DC-AC converters with realtime phase-lead FIR filter," IEEE Trans. Circuits Syst. II, vol. 53, no. 8, pp. 768-772, Aug. 2006.

[8] A. García-Cerrada, O. Pinzón-Ardila, V. Feliu-Batlle, P. RonceroSánchez, and P. García-González, "Application of a repetitive controller for a three-phase active power filter," IEEE Trans. Power Electron., vol. 22, no. 1, pp. 237-246, Jan. 2007.

[9] R. R. Ramos, D. Biel, E. Fossas, and F. Guinjoan, "A fixed-frequency quasi-sliding control algorithm: application to power inverters design by means of FPGA implementation," IEEE Trans. Power Electron., vol. 18, no. 1, pp. 344-355, Jan. 2003

[10] S. C. Tan, Y. M. Lai, C. K. Tse, and M. K. H. Cheung, "A fixedfrequency pulsewidth modulation based quasi-sliding-mode controller for buck converters," IEEE Trans. Power Electron., vol. 20, no. 6, pp. 1379-1392, Nov. 2005

[11] K. Zhang, Y. Kang, J. Xiong, and J. Chen, "Direct repetitive control of SPWM inverter for UPS purpose," IEEE Trans. Power Electron., vol. 18, no. 3, pp. 784-792, May. 2003.

[12] K. Srinivasan and F. R. Shaw, "Analysis and design of repetitive control systems using the regeneration spectrum," ASME J. Dynam. Syst. Meas. Control, vol. 113, no. 2, pp. 216-222, Jun. 1991.

[13] C. A. Desoer and M. Vidyasagar, Feedback Systems: Input-Output Properties. New York: Academic Press, 1975.

[14] DS1103 Hardware Installation and Configuration, dSPACE GmbH., Paderborn, 2005.

[15] ControlDesk Experiment Guide, dSPACE GmbH., Paderborn, 2006.

[16] RTI and RTI-MP Implementation Guide, dSPACE GmbH., Paderborn, 2006. 